\title{
Concurrent Abstract Machines
}

G Berry

Gentre de Mathematiques Appliquees, France

The notion of an abstract machine is widely used in sequential programming. Turing machines help in understanding effectivity and complexity issues. The SECD machine, the Categorical Abstract Machine or the Krivine machine implonent lambda-calculi, etc. So far, the abstract machine approach has not been applied to concurrent computations.

We present two concurrent abstract machines the Electrical Abstract Machine (ELAM) and the Chemical Abstract Machine (CHAM). The ELAM is a fixpoint equation solving machine based on a perfect synchrony hypothesis. It is a natural support for synchronous languages such as ESTEREL or LUSTRE. It is also akin to classical synchronous circuits. The CHAM is based on a chemical paradigm: computing agents are seen as molecules floating in a solution and interacting when they come in contact. We discuss the possible uses of such abstract machines. 